Canad. Math. Bull. Vol. 23 (2), 1980

\title{
ON THE CONNECTEDNESS OF CERTAIN SETS IN SUMMABILITY THEORY
}

\author{
BY \\ MANGALAM R. PARAMESWARAN
}

\begin{abstract}
This note considers the question of the connectedness of the set of limit points of the A-transforms of a sequence, where $A$ is a conservative Hausdorff, quasi-Hausdorff or Meyer-KönigRamanujan type of matrix. New proofs of some known results, as well as some new results are obtained.
\end{abstract}

$\S 1$

Given a conservative matrix $A$ and a sequence $s$, let $L(A ; s)$ denote the set of all limit points of the $A$-transform of $s$ (if it exists). Several authors have dealt with the question: "When is $L(A, s)$ connected for all $s \in(m)$, the space of bounded sequences?" and considered the cases where $A$ was Hausdorff or was quasi-Hausdorff. (See [1], [2], [3], [8] and also [4].) In the present paper we deal with the same question, and to certain refinements of it; we consider not only Hausdorff and quasi-Hausdorff matrices but also the Meyer-KönigRamanujan type of matrices $\left(S^{*}, \mu\right)$ introduced by Ramanujan [7]; we adopt a unified and somewhat novel approach which enables us to deal with matrices $A=(H, \mu)$ or $\left(H^{*}, \mu\right)$ or $\left(S^{*}, \mu\right)$ more or less simultaneously. The results obtained include and often improve the results obtained by the earlier authors, or are new.

We follow mainly the notation and definitions of Ramanujan [7] and Parameswaran [5], [6]. The proofs of our theorems are based on the following lemmas.

LEMMA 1. If $s=\left\{s_{n}\right\}$ is a bounded sequence and $a_{n} \equiv s_{n}-s_{n-1}=o(1)$, then $L(I, s)$ is connected. $(I=$ identity.) (Barone [1].)

Lemma 2. (See Parameswaran [5], pp. 52, 56 and 60; Cf. Ramanujan [7], pp. 205, 207 and 211.) Let $A=(H, \mu),\left(H^{*}, \mu\right)$ or $\left(S^{*}, \mu\right)$ be conservative. Then Canada.

Received by the editors April 21, 1976 and, in revised form, January 19, 1978.

* This work was completed when the author held an Operating Grant from the NRC of 
there exists a function $g \in B V[0,1]$ such that

$$
u_{n}=\int_{0}^{1} F_{n}(t, s) d g(t)=\left(\int_{0}^{0+}+\int_{0+}^{1-0}+\int_{1-0}^{1}\right) d g(t)
$$

where $u=\left\{u_{n}\right\}=A s$, and $\left\{F_{n}(t, s)\right\}$ denotes the Euler-transform $\left\{E_{n}(t, s)\right\}$, of order $t$, of $s$ or the 'Taylor-transform' $\left\{T_{n}(t, s)\right\}$ of $s$ and $s_{n}=O(1)$ or the 'MeyerKönig-transform $\left\{L_{n}(t, s)\right\}$ of $s$ and $s_{n}=O(1)$, according as $A=(H, \mu)$ or $\left(H^{*}, \mu\right)$ or $\left(S^{*}, \mu\right)$, respectively; further, if $A=\left(H^{*}, \mu\right)$ or $\left(S^{*}, \mu\right)$, the function $g(t)$ can be chosen so as to be continuous at $t=0$.

Lemma 3. Let A be a conservative Hausdorff or quasi-Hausdorff matrix. Then there exists a function $g \in B V[0,1]$ such that, with the notation $u=A s$,

$$
\text { (i) } u_{n}=\lambda[g(0+)-g(0)] s_{0}+\ell[g(1-0)-g(0+)]+s_{n}[g(1)-g(1-0)]+o(1)
$$

for all bounded sequences s Borel-summable to $\ell$, and with $\lambda=1$ or 0 according as $A$ is Hausdorff or not;

$$
\text { (ii) } u_{n}-u_{n-1}=\ell\left(\mu_{1}-\lim \mu_{n}\right)+\left(s_{n}-s_{n-1}\right) \lim \mu_{n}+o(1)
$$

if $A$ is Hausdorff and $\left\{s_{n}-s_{n-1}\right\}$ is bounded and Borel-summable to $\ell$, or if $s_{n}=O(1)$ and $A$ is Hausdorff or quasi-Hausdorff and then indeed (3) holds with $\ell=0$.

Lemma 3 is essentially contained in [5]; a proof is sketched below for completeness. Part (i) is proved by letting $n \rightarrow \infty$ in the righthand side of (1); note that under the conditions stated, $\left|F_{n}(t, s)\right| \leq K<\infty$ uniformly in $n$ and $t$, $\lim _{n \rightarrow \infty} F_{n}(t, s)=\ell(0<t<1)$,

$$
\lim _{t \rightarrow 0+} F_{n}(t, s)=\lambda s_{0} \quad \text { and } \quad \lim _{t \rightarrow 1-0} F_{n}(t, s)=s_{n} .
$$

For part (ii) of Lemma 3, we see that by Lemma 2,

$$
\begin{gathered}
u_{n}-u_{n-1}=\int_{0}^{1}\left[F_{n}(t, s)-F_{n-1}(t, s)\right] d g(t) \\
=\left(\int_{0}^{0+}+\int_{0+}^{1-0}+\int_{1-0}^{1}\right) d g(t)
\end{gathered}
$$

where (a) if $A$ is Hausdorff and $\left\{a_{n}\right\}=\left\{s_{n}-s_{n-1}\right\}$ is bounded and Borelsummable to $\ell$, the integrand in (4) reduces to $t E_{n-1}(t ; a)$, is uniformly bounded in $0 \leq t \leq 1$, tends to $\ell t$ for $0<t<1$ as $n \rightarrow \infty$, and for fixed $n$, tends to $a_{n}$ as $t \rightarrow 1-0$ and to 0 as $t \rightarrow 0+$; and (b) if $s_{n}=O(1)$, the integrand in (4) is uniformly bounded in $0 \leq t \leq 1$, tends to 0 as $n \rightarrow \infty$ for $0<t<1$ and, for fixed $n$, tends to $\lambda s_{0}$ as $t \rightarrow 0+$ and to $s_{n}-s_{n-1}$ as $t \rightarrow 1-0$. Then, in each of the cases 
(a) and (b), we get the desired result upon letting $n \rightarrow \infty$ in the righthand side of (4).

In this section the symbol $A$ may denote equally a conservative Hausdorff matrix $\left(H, \mu_{n}\right)$ or a conservative quasi-Hausdorff matrix $\left(H^{*}, \mu_{n}\right)$ except when explicitly specified.

THEOREM 1. (a) If $s \in(B)(m)$ [i.e. $s$ is a Borel-summable bounded sequence], then $L(A, s)$ is connected if $L(I, s)$ is connected.

(b) If $s \in(B)(m)$ and $L(A, s)$ is connected for some $A$ with $\lim \mu_{n} \neq 0$, then $L(I, s)$ is connected.

(c) If $s \notin(B)(m)$, then $L(A, s)$ is connected for some $A$ with $\lim \mu_{n} \neq 0 \not L L(I, s)$ is connected.

(c)'. In (c) above we may replace the phrase $\lim \mu_{n} \neq 0$ by $\lim \mu_{n}=0$.

(d) $\lim \mu_{n} \neq 0 \Leftrightarrow L(A, s)$ is connected for (only) almost no sequence of 0 's and 1 's $\Leftrightarrow L(A, s)$ is not connected when $s_{n}=\frac{1}{2}\left[1+(-1)^{n}\right]$.

Proof. Parts (a) and (b) follow from Lemmas 1 and 3(i). Part (c) is proved by the example given in Remark (iii) below; (c)' is a consequence of the fact that if $\lim \mu_{n}=0$ then $L(A, s)$ is connected for all $s \in(m)$, a known result which is also included in each of Theorems 2 and 3 below. Part (d) follows from parts (a) and (b), since almost all sequences of 0's and 1's, and in particular $\left\{\frac{1}{2}+\frac{1}{2}(-1)^{n}\right\}$, are Borel-summable.

Remarks. (i) Theorems 1(a), 1(b) may be compared with earlier results of Ramanujan ([17], Theorems 5, 7) and of the author ([6], theorem 2(i), (ii)) and Theorem 1(d) with another result of the author ([5], Theorem 10) which deal with the A-summability of $s$, i.e. the case when $L(A, s)$ has a unique element. (See also the remark under Theorem 4 below.)

(ii) Theorem 1 (a) has non-trivial content. For, it is known that if we take any function $F(n) \neq o\left(n^{-1 / 2}\right)$ with $F(n) \downarrow 0$, e.g. $F(n)=n^{-1 / 4}$, then there exist Borel-summable bounded divergent sequences $s$ with $s_{n}-s_{n-1}=O(F(n))=$ $o(1)$. By Lemma $1, L(I, s)$ is connected, and hence so is $L(A, s)$ by Theorem 1(a). Thus there are Borel-summable bounded divergent sequences $s$ for which $L(A, s)$ is connected for every conservative $A=(H, \mu)$ or $\left(H^{*}, \mu\right)$. (See also the corollary to Theorem 3 below.)

(iii) Theorem 1(c) shows that Theorem 1(b) is a best possible one. Theorem 1(d) is also a best possible result in the sense that there exists even a regular Hausdorff matrix $A=\left(H, \mu_{n}\right)$ with $\lim \mu_{n} \neq 0$ and a divergent sequence $s$ of 0 's and 1's such that $L(A, s)$ is connected, and there exists also a regular quasiHausdorff matrix $G=\left(H^{*}, \nu_{n}\right)$ with $\lim \nu_{n} \neq 0$ and a divergent sequence $t$ of 0 's 
and 1 's such that $L(G, t)$ is connected, while obviously, neither $L(I, s)$ nor $L(I, t)$ is connected.

To see this we take $A=\frac{1}{2}\left(I+C_{1}\right)$, where $C_{1}$, is the Cesaro matrix, and $s$ to be a sequence consisting of alternating bunches of 0 's and 1's as follows: $s_{0}=1$, $s_{n}=0\left(n_{2 k}<n \leq n_{2 k+1}\right), s_{n}=1\left(n_{2 k+1}<n \leq n_{2 k+2}\right)$, where $\left\{n_{k}\right\}$ is a sequence of positive integers which increases so rapidly that $L(A, s)$ consists of the interval $[0,1]$; for instance, we may take $n_{k}=\exp _{10}\left(2^{k}\right)$.

For the quasi-Hausdorff case, we take $G=\frac{1}{2}\left(I+C_{1}^{*}\right)$, where $C_{1}^{*}=\left(H^{*}, \mu_{n}\right)$ with $\mu_{n}=1 /(n+2)$. It is then not difficult to see that there exists a divergent sequence $t$ of 0 's and 1 's such that $L(G, t)$ is the interval $[0,1]$.

(iv) Leviatan and Lorch [3] raised the following question: Do the limit points of equivalent transforms of bounded sequences have the same connectedness properties? The matrix $A=\frac{1}{2}\left(I+C_{1}\right)$ considered above is equivalent to $I$ for all sequences, and since $L(A, s)$ is connected while $L(I, s)$ is not (where $s$ is as defined in (iii) above), it is seen that the question is answered in the negative for Hausdorff matrices; similarly, for quasi-Hausdorff matrices, the matrices $G$ and $I$ and the sequence $t$ mentioned in (iii) above again provide an answer in the negative.

(v) The examples $u=A s$ and $v=G t$, where $A, G, s$ and $t$ are as in Remark (iii) above, provide yet other proofs of the known fact that the converse of Lemma 1 is not true.

THEOREM 2. The following statements are equivalent:

(a) $\lim \mu_{n}=0$;

(b) $L(A, s)$ is connected for some Borel-summable divergent sequences of 0's and 1's;

(c) $L(A, s)$ is connected for all bounded sequences $s$;

(d) A sums a Borel-summable bounded divergent sequence;

(e) A sums all Borel-summable bounded sequences.

If $A$ is Hausdorff, then each of the following is also equivalent to (a):

(f) $L(A, s)$ is connected for all sequence $s=\left\{s_{n}\right\}$ for which As $\in(m), s_{n}=$ $o\left(n^{1 / 2}\right)$ and $s_{n}-s_{n-1}=O(1)$;

(g) $L(A, s)$ is connected for all sequences $s=\left\{s_{n}\right\}$ for which $A s \in(m)$ and $\left\{s_{n}-s_{n-1}\right\}$ is bounded and Borel-summable to 0 .

Proof. it is well-known that $(\mathrm{a}) \Leftrightarrow(\mathrm{d}) \Leftrightarrow(\mathrm{e})$ (Parameswaran [6], Theorem 2); (c) $\Rightarrow(b)$, trivially; (b) $\Rightarrow$ (a) by Theorem 1(b), and the implication (a) $\Rightarrow$ (c) follows from Lemmas 1 and 3(ii).

If $A$ is Hausdorff, then (a) $\Rightarrow(\mathrm{g}) \Rightarrow$ (f) by Lemmas 1 and 3(ii) and the fact that if $s_{n}=o\left(n^{1 / 2}\right)$ then $\left\{s_{n}-s_{n-1}\right\}$ is Borel-summable to 0 ; since (f) $\Rightarrow$ (c) trivially, it follows, from the equivalences already proved that all of $(\mathrm{a})-(\mathrm{g})$ are equivalent. 
Remarks. (i) For regular Hausdorff methods, the relation (a) $\Leftrightarrow$ (c) was given by Wells [8] and Erdös and Piranian [2]; Leviatan and Lorch [3] showed that the relation (c) $\Rightarrow$ (a) holds for multiplicative Hausdorff matrices, and that (c) $\Leftrightarrow$ (a) for conservative quasi-Hausdoff matrices. (ii) The examples considered in Remark (iii) under Theorem 1 show that if the word "Borel-summable" is dropped from the statement (b) of Theorem 2 then the theorem will be true neither for Hausdorff nor for quasi-Hausdorff matrices. (iii) See also the concluding remarks at the end of the paper.

The next two theorems follow readily from Lemmas 1 and 3(ii) and yield conditions that are sufficient in order that $L(A, s)$ be connected for a given pair $A$ and $s$.

THEOREM 3. Let $s \in(m)$ and $A$ be given. Then $L(A, s)$ is connected if $\mu_{n} a_{n} \equiv \mu_{n}\left(s_{n}-s_{n-1}\right)=o(1)$.

COROllary. There exist bounded divergent sequences $s$ which are not Borelsummable and such that $L(A, s)$ is connected for every conservative $A=(H, \mu)$ or $\left(H^{*}, \mu\right)$. (Cf. Remark (ii) under Theorem 1.)

For, we can take any bounded sequence $\left\{s_{n}\right\}$ which is not Borel-summable and for which $s_{n}-s_{n-1}=o(1)$.

ThEOREM 4. Let $A=(H, \mu)$ be conservative Hausdorff and $\left\{s_{n}\right\}$ a sequence that $A s \in(m)$ and $\left\{a_{n}\right\} \equiv\left\{s_{n}-s_{n-1}\right\}$ is bounded and Borel-summable to $\ell$. Then $L(A, s)$ will be connected if any one of the following conditions holds:

(i) $a_{n}=o(1)$;

(ii) $a_{n} \rightarrow \ell$ and $\mu_{1}=0$;

(iii) $\mu_{n} \rightarrow \ell=0$;

(iv) $\mu_{n} \rightarrow \mu_{1}=0$.

Remark. The example of $A$ and $s$ given in Remark (iii) under Theorem 1 show that none of the conditions given in Theorems 3 or 4 is necessary for $L(A, s)$ to be connected. However, Theorem 3 is best possible in the sense that we cannot replace the small $o$ by a large $O$, as is seen from Theorem 1(d).

In an earlier paper (Parameswaran [5], Theorem 8) it is proved that a conservative matrix $A=\left(S^{*}, \mu\right)$ of the Meyer-König-Ramanujan type sums all Borel-summable bounded sequences, irrespective of whether $\lim \mu_{n}$ is 0 or not. The contrast with the Hausdorff and quasi-Hausdorff matrices is reflected also in the following result.

THEOREM 5. If $A=\left(S^{*}, \mu\right)$ is conservative, then $L(A, s)$ is connected for all bounded sequences $s$. 
Proof. If $u=A s$ where $A, s$ are as in the theorem, then

$$
u_{n} \int_{0+}^{1-0} L_{n}(t, s) d g(t)+s_{0}[g(1)-g(1-0)]
$$

by (1), since $\lim _{t \rightarrow 1-0} L_{n}(t, s)=s_{0}$; hence

$$
u_{n}-u_{n-1}=\int_{0+}^{1-0}\left[L_{n}(t, s)-L_{n-1}(t, s)\right]=o(1)
$$

since the integrand is uniformly bounded and tends to 0 as $n \rightarrow \infty$ for $0<t<1$; (these are given in [5], p. 60 and p. 51 respectively). The theorem now follows from Lemma 1.

Concluding remarks. The Authors thanks the referee for drawing his attention to Liu and Rhoades [4] which appeared after the first version of the present paper was submitted. Liu and Rhoades consider the regular 'generalized' Hausdorff and quasi-Hausdorff matrices $\left(A^{(\alpha)}, \mu_{n}\right)=\left(H^{(\alpha)}, \mu_{n}\right)$ or $\left(H^{*(\alpha)}, \mu_{n}\right)$ for $\alpha \geq 0$. However each conservative generalized matrix $A^{(\alpha)}$, whether it is a matrix of one of the above two types or is a 'generalized' MeyerKönig-Ramanujan matrix $\left(S^{*(\alpha)}, \mu_{n}\right)$ is absolutely equivalent for bounded sequences to an ordinary matrix of the same kind. (This result and some others on the $\left(A^{(\alpha)}, \mu\right)$-matrices will appear elsewhere.) The theorems of the present paper are therefore true also for the generalized matrices $A=\left(H^{(\alpha)}, \mu\right)$ or $\left(H^{*(\alpha)}, \mu\right)$ or $\left(S^{*(\alpha)}, \mu\right), \alpha \geq 0$; thus they include, and extend, the results of Liu and Rhoades [4].

Finally, the author thanks the referee for many comments which improved the presentation of the results.

\section{REFERENCES}

1. H. G. Barone: Limit points of sequences and their transforms by methods of summability, Duke Math. J. 5 (1939), 740-752.

2. P. Erdös and G. Piranian: Laconicity and redundancy of Toeplite matrices, Math. Zeit 83 (1964), 381-394.

3. D. Leviatan and L. Lorch: On the connectedness of the sets of limit points of certain transforms of bounded sequences. Canad. Math. Bull. 14 (1971), 175-181.

4. M. Liu and B. E. Rhoades: Some properties of generalized Hausdorff matrices, Houston J. Math. 2 (1976), 239-250.

5. M. R. Parameswaran: Some remarks on Borel summability, Quart. J. Math. (Oxford) (2) 10 (1959), 224-229.

6. M. R. Parameswaran: on the translativity of Hausdorff-and some related methods of summability, J. Indian Math. Soc. 23 (1959), 45-64.

7. M.. S. Ramanujan: On Hausdorff and quasi-Hausdorff methods of summability, Quart. J. Math. (Oxford) (2) 8 (1957), 197-213.

8. J. H. Wells: Hausdorff transforms of bounded sequences, Proc. American Math. Soc. 11 (1960), 84-86.

Dept. of Mathematics AND Astronomy

THE UNIVERSITY OF MANITOBA

WINNIPEG, R3T 2N2 